\title{
Comparison of angular frequency contrast sensitivity in young and older adults
}

\section{M.L.B. Simas ${ }^{2}$ and R.M.T.B.L. Nogueira ${ }^{2}$}

N.A. Santos ${ }^{1}$,
${ }^{1}$ Departamento de Psicologia, Universidade Federal da Paraíba,

Jõao Pessoa, PB, Brasil

${ }^{2}$ Departamento de Psicologia, Universidade Federal de Pernambuco, Recife, PE, Brasil

\begin{abstract}
Correspondence
N.A. Santos

Departamento de Psicologia

CCHLA, UFPB

58059-900 João Pessoa, PB

Brasil

Fax: +55-83-216-7064

E-mail: natanael@ufpe.br or

natanael@cchla.ufpb.br

Research supported by $\mathrm{CNPq}$ and FACEPE.
\end{abstract}

Received May 8, 2003

Accepted December 4, 2003

\begin{abstract}
The aim of the present study was to measure contrast sensitivity curves for angular frequencies in the range between 2 and 96 cycles $/ 360^{\circ}$ in older human adult volunteers and to compare these measurements with the more usual contrast sensitivity functions for sine-wave gratings. All subjects were free of identifiable ocular disease and had normal acuity. We measured the contrast thresholds for young adults $(\mathrm{N}=6$; age range, 20-26 years) and older adults $(\mathrm{N}=6$; age range, 60 67 years) using the psychophysical forced-choice method. In this paradigm the volunteers had to choose the stimulus containing a test frequency at low contrast (e.g., either a sine-wave grating or an angular frequency stimulus), or another neutral stimulus at mean luminance (without any contrast). Older adults presented a loss in contrast sensitivity at high and medium angular frequencies compared to the young adults (i.e., from 8 to 96 cycles $/ 360^{\circ}$ ). Contrary to expectation, contrast sensitivity at low angular frequencies, i.e., 2 and 4 cycles/ $360^{\circ}$, was better for the older group than for the younger group. On the other hand, contrast sensitivity for sine-wave gratings at 3 and $4 \mathrm{cpd}$ was higher for young adults as expected. These results suggest agerelated changes in the contrast sensitivity function for angular frequencies.
\end{abstract}

The characteristic neural and optical changes occurring in the aging human visual system have been the subject of a variety of studies relating them to the contrast sensitivity function (CSF) (1-7). Although there is no consensus about the frequency ranges affected by aging, the main findings are: i) moderate decreases in sensitivity for medium and high spatial frequencies as indicated by the CSF $(3,7,8)$, and ii) the most significant decreases in the CSF occur only for
Key words

- Human aging

- Spatial frequency

- Angular frequency

- Contrast sensitivity

- Forced-choice method

$\ldots \ldots \ldots \ldots \ldots \ldots$ adults over 50 years old $(5,9,10)$.

In the present study we measured the CSF for angular frequency stimuli (aCSF) in young and older adults using a forced-choice paradigm. Angular frequencies are spatial frequency stimuli defined in terms of polar coordinates (see further details in Ref. 11). They are referred to in the literature as windmill, radial stimuli or polar gratings (11). Contrary to sine-wave gratings, angular frequency stimuli are independent of observa- 
tion distance.

Our objective was to relate the aging process to the aCSF. Studies on human vision measuring sensitivity to angular frequencies are rare for young adults $(11,12)$ and non-existent for the older population. Six young adults (20-26 years old) and six older adults (60-67 years old) were studied in the present investigation. They were free of ocular diseases and had been examined by an ophthalmologist over the last 12 months. They had normal or corrected to normal visual acuity.

Stimuli were generated on a SONYBVM-1910 monitor, with RGBsync input controlled by a microcomputer through a DT-2853 frame grabber. Measurements were made at a distance of $150 \mathrm{~cm}$ from the monitor screen. A neutral gray plate was placed above the monitor to be fixated upon between trials to minimize visual after effects. Mean luminance was $6.9 \mathrm{~cd} / \mathrm{m}^{2}$ measured by an Asahi Pentax $1^{\circ}$ spot meter. The minimum and maximum luminance values were 6.2 and $7.5 \mathrm{~cd} / \mathrm{m}^{2}$. The room was gray for better control of ambient luminance.

The achromatic angular frequencies were $2,4,8,16,24,32,48,64$ and 96 cycles $/ 360^{\circ}$. The aperture was circular and had a diameter of 7.25 degrees of visual angle (Figure 1).

Measurements were made using a temporal two-alternative forced-choice paradigm $(11,12)$. This paradigm is based on the prob-

Figure 1. Examples of angular frequency stimuli at 2, 4, 8 and 16 cycles $/ 360^{\circ}$. Angular frequency stimuli are integer and distance independent.

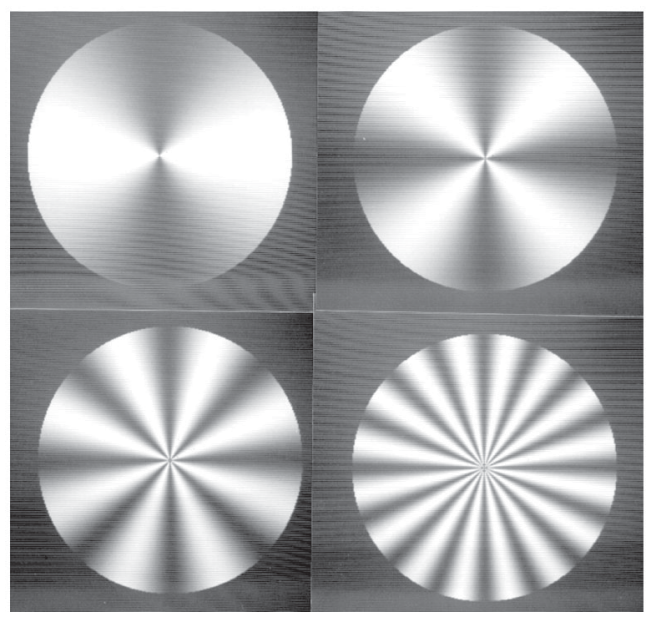

ability of consecutive correct choices made by the subjects following the presentation of two successive stimuli, in this case, one at mean luminance $\left(6.9 \mathrm{~cd} / \mathrm{m}^{2}\right)$ and the other containing one of the angular frequencies. A correct choice was to select the angular frequency stimuli (first or second of each pair). The number of consecutive correct answers required for lowering contrast by a unit $(0.08 \%)$ was set at three. Contrast was always increased by the same unit after each incorrect choice. This yielded a probability of $79 \%$ for perceiving the angular frequency stimuli.

An experimental session started with a brief beep followed by the presentation of one of the stimuli for $2 \mathrm{~s}$, followed by an interstimulus interval lasting $1 \mathrm{~s}$ and by the presentation of the other stimulus for $2 \mathrm{~s}$, followed by the response. The intertrial interval was $3 \mathrm{~s}$. A different beep provided the feedback about each correct answer. The duration of an experimental session depended on the responses given by the subject and ended only after 10 pairs ( 5 pairs for the older adults) of peaks and valleys were obtained (i.e., 20-point estimate values, or 10 for the older adults). The session generally lasted about 15-20 min.

Each point estimate was measured at least twice, always on different days, for each subject. A total of twelve functions (of 9point estimates each) was measured for both groups of subjects, yielding a sample of about 120- to 240-point estimates for each angular frequency being measured.

Figure 2 shows the angular frequency contrast threshold function (1/aCSF) for young and older adults. Contrast threshold estimates are shown as a function of angular frequencies. The standard error of the mean (error bars) was corrected for the size of the sample to represent the $99 \%$ confidence interval.

The functions (1/aCSF) for both groups show that maximum sensitivity occurred at 16 and 32 cycles $/ 360^{\circ}$, i.e., at medium angu- 
lar frequencies. Older adults needed 0.6 times more contrast than the young adult group at medium and high angular frequencies (Figure $2 \mathrm{~A}$ ). On the other hand, for very low angular frequencies (i.e., 2 and 4 cycles) the older group needed less contrast. In the aCSF, all differences between groups were statistically significant (i.e., $\mathrm{P}<0.000001$ for both 2 cycles and 4 cycles; $t$-test for independent samples).

The present results show that sensitivity to angular frequencies is better for young adults at medium and high angular frequencies compared to older adults, while sensitivity at low and very low angular frequencies is better for the older group.

Our main objective was to characterize the visual systems of young and older adults by measuring the aCSF. We wanted to evaluate the possible processing changes as the result of aging. The results showed that this function is different for young (20-26 years old) and older adults (60-67 years old), with a decrease in sensitivity for older adults at medium and high frequencies, similar to that found for spatial frequencies in the literature $(5,7,9,13-15)$. However, the higher sensitivity found for the older group in the range of $2-4$ cycles $/ 360^{\circ}$ was unexpected. A $t$-test for independent samples showed $\mathrm{P}<0.000001$ for both 2 cycles $/ 360^{\circ}$ and 4 cycles $/ 360^{\circ}$. There are no studies in the literature reporting this finding. On the contrary, several studies have reported a decline also at low spatial frequencies $(6,8,16)$.

In order to determine whether this difference was due to the experimental procedure, equipment or paradigm used, we also measured the CSF for spatial frequencies with sine-wave gratings (i.e., $0.5,1.0,2.0,3.0$, 4.0, 6.0 and 9.0 cycles per degree of visual angle, cpd) for the same subjects, same size, same method and equivalent experimental conditions (Figure 2B). The results for the $\mathrm{CSF}$ are similar to those in the literature for sine-wave gratings, showing lower sensitivity for the older adults at medium and high
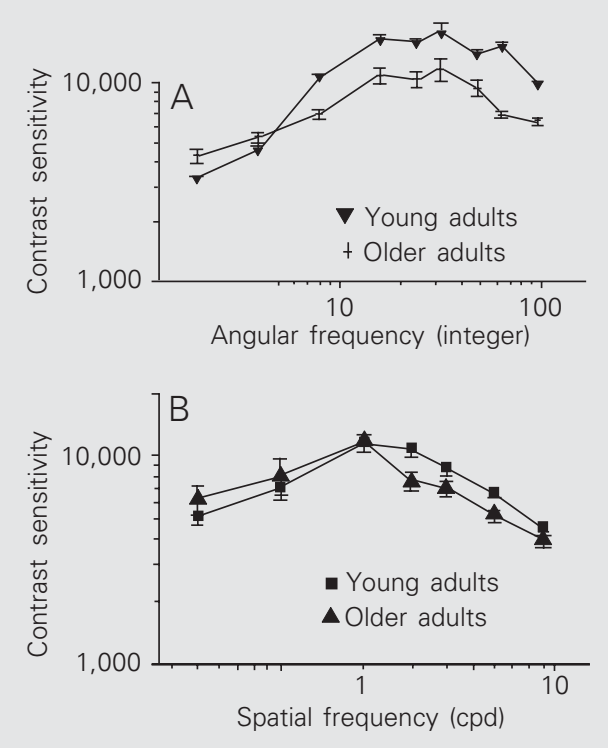

Figure 2. Angular frequency sensitivity functions for young and older adults (A) and spatial frequency contrast sensitivity for vertical sine-wave gratings for the two groups (B). Reported data are the grand mean for 6 individuals in each group. spatial frequency (1-9 cpd). In this case, we found no improvement at low frequencies when sine-wave gratings were used. Figure 2B shows the CSF for young and older adults. Under these conditions, only spatial frequencies of 3.0 and $4.0 \mathrm{cpd}$ showed significant differences (i.e., using a $t$-test for independent samples, $\mathrm{P}=0.000001$ for both spatial frequencies).

Thus, the improvement at low angular frequency (i.e., at 2 and 4 cycles $/ 360^{\circ}$ ) observed for the older adults when compared to the younger group may be related to differences of the channels, putative mechanisms involved in the processing of angular frequencies and sine-wave gratings. The available literature suggests that these stimuli (i.e., angular frequency and sine-wave gratings) are most likely processed by different visual cortical areas $(12,17-19)$. It is too soon to state that aging affects the underlying putative mechanisms for spatial and angular frequencies in different ways, particularly at lower angular frequencies, but some studies have suggested that aging alters the visual pathways $(3,6,8,17,20)$. Since these are rather unexpected results we think they require further investigation. 


\section{References}

1. Arden GB \& Jacobsen J (1978). A simple grating test for contrast sensitivity: preliminary results indicate value for screening in glaucoma. Investigative Ophthalmology and Visual Science, 17: 23-32.

2. Arundale $K$ (1978). An investigation into the variation of human contrast sensitivity with age and ocular pathology. British Journal of Ophthalmology, 62: 213-215.

3. Elliott D, Whitaker D \& MacVeigh D (1990). Neural contribution to spatiotemporal contrast sensitivity decline in healthy ageing eyes. Vision Research, 30: 541-547.

4. McGrath C \& Morrison JD (1981). The effects of age on spatia frequency perception in human subjects. Quarterly Journal of Experimental Physiology, 66: 253-261.

5. Owsley C, Sekuler R \& Siemsen D (1983). Contrast sensitivity throughout adulthood. Vision Research, 23: 689-699.

6. Sekuler R, Hutman LP \& Owsley C (1980). Human aging and spatial vision. Science, 209: 1255-1256.

7. Fiorentini A, Porciatti V, Morrone MC \& Burr DC (1996). Visual ageing: Unspecific decline of the responses to luminance and colour. Vision Research, 36: 3557-3566.

8. Schefrin BE, Tregear SJ, Harvey Jr LO \& Werner JS (1999). Senescent changes in scotopic contrast sensitivity. Vision Research, 39: 3728-3736.

9. Higgins KE, Jaffe MJ, Caruso RC \& deMonasterio FM (1988). Spatial contrast sensitivity: effects of age, test-retest, and psychophysical method. Journal of the Optical Society of America. A, Optics, Image Science, and Vision, 5: 2173-2180.

10. Nio YK, Jansonius NM, Fidler V, Geraghty E, Norrby S \& Kooijman AC (2000). Age-related changes of defocus-specific contrast sensitivity in healthy subjects. Ophthalmic and Physiological Optics, 20: 323-334.

11. Simas MLS, Santos NA \& Thiers FA (1997). Contrast sensitivity to angular frequency stimuli is higher than that for sine wave gratings in the respective middle range. Brazilian Journal of Medical and Biological Research, 30: 633-636.

12. Simas MLS \& Santos NA (2002). Narrow-band 1, 2, 3, 4, 8, 16 and 24 cycles $/ 360^{\circ}$ angular frequency filters. Brazilian Journal of Medical and Biological Research, 35: 243-253.

13. Crassini B, Brown B \& Bowman D (1988). Age-related changes in contrast sensitivity in central and peripheral retina. Perception, 17: 315-332.

14. Elliott DB (1987). Contrast sensitivity decline with ageing: A neural or optical phenomenon? Ophthalmic and Physiological Optics, 7: 415-419.

15. Ross JE, Clarke DD \& Bron AJ (1985). Effect of age on contrast sensitivity function: Uniocular and binocular findings. British Journal of Ophthalmology, 69: 51-56.

16. Kackson GR, Owsley C, Cordle EP \& Finley DF (1998). Aging and scotopic sensitivity. Vision Research, 38: 3655-3662.

17. Gallant JL, Connor CE, Rakshit S, Lewis JW \& van Essen DC (1996). Neural responses to polar, hyperbolic, and cartesian gratings in area V4 of the macaque monkey. Journal of Neurophysiology, 76: 27182739.

18. Merigan WH (1996). Basic visual capabilities and shape discrimination after lesions of extrastriate area V4 in macaques. Visual Neuroscience, 13: 51-60

19. Wilkinson F, James TW, Wilson HR, Gati JS, Menon ES \& Goodale MA (2000). An fMRI study of the selective activation of human extrastriate form vision areas by radial and concentric gratings. Current Biology, 10: 1455-1458.

20. Habak C \& Faubert $J$ (2000). Larger effect of aging on the perception of higher-order stimuli. Vision Research, 40: 943-950. 